\title{
Correction to: Population pharmacokinetics of trastuzumab emtansine in previously treated patients with HER2-positive advanced gastric cancer (AGC)
}

\author{
Shang-Chiung Chen ${ }^{1} \cdot$ Matts Kagedal $^{1} \cdot$ Yuying Gao ${ }^{2} \cdot$ Bei Wang $^{1} \cdot$ Marie-Laurence Harle-Yge ${ }^{3}$. Sandhya Girish ${ }^{1}$. \\ Jin Jin ${ }^{1}$. Chunze Li
}

Published online: 30 November 2017

(c) Springer-Verlag GmbH Germany, part of Springer Nature 2017

\section{Correction to:}

Cancer Chemother Pharmacol (2017) 80:1147-1159 https://doi.org/10.1007/s00280-017-3443-1

The author would like to correct the errors in the publication of the original article. The corrected details are given below for your reading.

In the "Introduction" section, last sentence of the second paragraph should read as:

The estimated clearance, volume of distribution of the central compartment $(\mathrm{Vc})$, and terminal elimination halflife of T-DM1 were $0.676 \mathrm{~L} /$ day, $3.127 \mathrm{~L}$ and 3.94 days, respectively [13].
In the "PK sampling and bioanalytic method" section, Third sentence should read as:

For the T-DM1 $2.4 \mathrm{mg} / \mathrm{kg}$ qw regimen, additional PK samples were collected at end of infusion on day 8 and day 15 of Cycle 1. Post-T-DM1 regimen-selection analysis, PK samples were collected at predose and end of infusion of Cycle 1 and 4 (day 1); predose Cycle 2. Patients were defined as evaluable for PopPK analysis if they had at least one adequately documented dose of T-DM1 and a corresponding PK sample.

Table 1 should read as:

The original article can be found online at https://doi. org/10.1007/s00280-017-3443-1.

Shang-Chiung Chen

chen.shangchiung@gene.com

1 Genentech, Inc., 1 DNA Way, South San Francisco, CA 94080, USA

2 Certara USA, 100 Overlook Center \#101, Princeton, NJ 08540, USA

3 F. Hoffmann-La Roche Ltd., 4070 Basel, Switzerland 
Table 1 Covariate values of GATSBY PopPK dataset

\begin{tabular}{|c|c|c|c|c|}
\hline & \multicolumn{2}{|c|}{$2.4 \mathrm{mg} / \mathrm{kg} \mathrm{qw}$} & \multicolumn{2}{|c|}{$3.6 \mathrm{mg} / \mathrm{kg} \mathrm{q} 3 \mathrm{w}$} \\
\hline & $N$ & Median (min, max) & $N$ & Median (min, $\max$ ) \\
\hline \multicolumn{5}{|l|}{ Continuous covariates } \\
\hline Age (years) & 101 & $61(22,78)$ & 35 & $64(24,78)$ \\
\hline BW (kg) & 101 & $63.0(35.2,99.6)$ & 35 & $58.8(42.5,94.9)$ \\
\hline CRCL (mL/min) & 97 & $74.5(29.3,175.0)$ & 35 & $74.2(25.6,120.2)$ \\
\hline SGOT (U/L) & 95 & $23(9,103)$ & 35 & $23(12,92)$ \\
\hline SGPT (U/L) & 96 & $16(5,67)$ & 35 & $16(5,57)$ \\
\hline TBIL $(\mu \mathrm{mol} / \mathrm{L})$ & 96 & $8.6(3.4,25.1)$ & 35 & $8.0(3.3,27.4)$ \\
\hline ALKP (U/L) & 95 & $129(42,596)$ & 35 & $130(42,613)$ \\
\hline PLAT $\left(\times 10^{9} / \mathrm{L}\right)$ & 93 & $230(107,595)$ & 34 & $227(123,853)$ \\
\hline $\mathrm{ECD}(\mathrm{ng} / \mathrm{mL})$ & 85 & $9.0(2.2,515.3)$ & 33 & $8.6(2.2,241.9)$ \\
\hline $\mathrm{TMBD}(\mathrm{mm})$ & 90 & $57(10,210)$ & 31 & $67(15,246)$ \\
\hline $\mathrm{TBL}(\mu \mathrm{g} / \mathrm{mL})$ & 98 & $0.65(0.00,42.50)$ & 35 & $0.00(0.00,38.70)$ \\
\hline \multicolumn{5}{|l|}{ Categorical covariates } \\
\hline Sex (male/female) & $75 / 26$ & & $26 / 9$ & \\
\hline Race (White/Asian/other) & $38 / 59 / 4$ & & $14 / 18 / 3$ & \\
\hline ECOG PS (0/1) & $49 / 52$ & & $13 / 22$ & \\
\hline Measurable disease (yes/no) & $90 / 11$ & & $31 / 4$ & \\
\hline Visceral disease (yes/no) & $61 / 40$ & & $20 / 15$ & \\
\hline Number of disease sites $(<3 / \geq 3)$ & $45 / 56$ & & $14 / 21$ & \\
\hline Cancer type (gastric/GEJ) & $72 / 29$ & & $25 / 10$ & \\
\hline $\begin{array}{l}\text { Cancer histologic subtype (intesti- } \\
\text { nal/diffuse/mixed/unknown) }{ }^{\mathrm{a}}\end{array}$ & $39 / 23 / 5 / 34$ & & $13 / 3 / 1 / 18$ & \\
\hline Prior gastrectomy (yes/no) & $30 / 71$ & & $11 / 24$ & \\
\hline Bone metastasis (yes/no) & $7 / 94$ & & $2 / 33$ & \\
\hline Liver metastasis (yes/no) & $49 / 52$ & & $15 / 20$ & \\
\hline Lung metastasis (yes/no) & $28 / 73$ & & $7 / 28$ & \\
\hline
\end{tabular}

$A L K P$ alkaline phosphatase concentration, $B W$ body weight, $C R C L$ creatinine clearance, $E C D$ serum human epidermal growth factor receptor 2 shed extracellular domain concentration, ECOG PS Eastern Cooperative Oncology Group performance status, GEJ gastroesophageal, PLAT baseline platelet count, $P O P P K$ population pharmacokinetics, SGOT serum aspartate aminotransferase concentration, $S G P T$ serum alanine aminotransferase concentration, $T B L$ baseline trastuzumab concentration, $T B I L$ total bilirubin concentration, TMBD tumor burden, $q w$ every week, $q 3 w$ every 3 weeks

${ }^{a}$ Per investigator assessment
In the "Discussion" section, tenth sentence should read as:

The interaction between trastuzumab and its target, the membrane-bound receptor HER2, may account for the nonlinear elimination pathway.

In the "Discussion" section, eleventh sentence of the fourth paragraph should read as:

GATSBY successfully addressed this concern and the regimen that was selected for further evaluation in the phase III portion of the study was $2.4 \mathrm{mg} / \mathrm{kg}$ qw.
Reference 6 should read as:

Kadcyla $^{\mathrm{TM}}$ (ado-trastuzumab emtansine) (2013) Prescribing information (USA). Genentech. http://www.accessdata.fda.gov/drugsatfda_docs/label/2013/125427lbl.pdf. Accessed June 2016. 